山्यRANÇAISE

$>\mathrm{DE}$

$\stackrel{1=1}{\simeq}$ PÉDAGOGIE

\section{Revue française de pédagogie}

Recherches en éducation

174 | janvier-mars 2011

Évaluation, développement professionnel et organisation scolaire

\title{
Associer obligation de résultats et amélioration des compétences des établissements de New York
}

Bringing together accountability and skills improvement in New York's schools Asociar obligación de resultados y mejora de las competencias de los establecimientos de Nueva York

Ergebnispflicht und Verbesserung der Kompentenzen an New-Yorker Schulen vereinbaren

\section{Marian Robinson}

\section{OpenEdition}

Journals

Édition électronique

URL : http://journals.openedition.org/rfp/2964

DOI : $10.4000 /$ rfp. 2964

ISSN : 2105-2913

Éditeur

ENS Éditions

Édition imprimée

Date de publication : 15 mars 2011

Pagination : 49-60

ISBN : 978-2-7342-1203-4

ISSN : 0556-7807

Référence électronique

Marian Robinson, "Associer obligation de résultats et amélioration des compétences

des établissements de New York », Revue française de pédagogie [En ligne], 174 I janvier-mars 2011,

mis en ligne le 15 mars 2015, consulté le 21 décembre 2020. URL : http://journals.openedition.org/rfp/ 2964 ; DOl : https://doi.org/10.4000/rfp.2964 


\title{
Associer obligation de résultats et amélioration des compétences des établissements de New York
}

\author{
Marian Robinson
}

L'un des défis auxquels sont confrontés les responsables politiques américains est de concevoir des systèmes d'obligation de résultats (ou accountability) et d'investir dans le renforcement des compétences des enseignants, pour in fine contribuer à l'amélioration des résultats des élèves. Children First, réforme ambitieuse lancée par la ville de New York, éclaire quelques-uns des choix stratégiques auxquels se trouvent confrontés les responsables politiques pour combiner objectifs de performance et amélioration de la qualité de l'enseignement et des apprentissages. L'article analyse ce double objectif afin de comprendre les liens entre la pression exercée par les politiques d'obligation de résultats et les nouvelles ressources mises à disposition pour renforcer les compétences des établissements scolaires. Children First fixait non seulement des objectifs de performance pour chaque établissement scolaire, mais il permettait aussi aux chefs d'établissement d'assurer un meilleur suivi des ressources, en même temps qu'il fournissait de nouveaux systèmes de données et d'évaluations formatives afin d'aider les enseignants à analyser la qualité de leur enseignement et des apprentissages des élèves. Par ailleurs, cette réforme instituait des équipes d'enquête collaborative dans chaque établissement et ouvrait l'accès à un nouveau marché de prestataires de services d'accompagnement et de développement professionnel. Deux ans après sa mise en place, l'article fait le point sur ce dispositif, notamment sur le processus d'enquête collaborative, essentiel à la mise en œuvre d'une organisation visant la production de connaissances et la mobilisation des ressources pour accompagner les élèves les plus en difficulté. Les données à l'appui de l'analyse sont issues d'entretiens et d'observations menés en 2008 dans 41 écoles publiques de la ville de New York.

Descripteurs (TESE) : responsabilisation, projet d'école, travail en équipe, qualité de l'enseignement, résultats de l'éducation, difficulté d'apprentissage.

\section{INTRODUCTION}

Le projet politique d'amélioration de l'école aux États-Unis est presque toujours associé à l'obligation de résultats (accountability) selon des critères de performance. Dans chaque État, ce projet poursuit deux objectifs à la fois : la recherche de l'équité et de l'excellence en éducation. Des standards de performance élevés sont assignés aux élèves dans les matières principales (l'anglais et les mathématiques entre autres) et tous les élèves doivent atteindre ces standards, même ceux qui ont de manière chronique le plus de difficultés. Le dispositif de première génération reposait sur des mesures incitatives complexes 
qui alliaient récompenses et sanctions pour motiver enseignants et élèves à atteindre ces objectifs. L'hypothèse de départ était que les objectifs seraient atteints si tous faisaient les efforts nécessaires et si les enseignants adaptaient leurs cours aux nouvelles exigences.

Les recherches sur les résultats de ce dispositif initial montrent bien ses limites (Fuhrman \& Elmore, 2004). Les établissements les moins performants ont réagi par une préparation plus intensive aux tests et non par un meilleur accès des élèves à un enseignement de qualité. Les responsables de ce dispositif n'étaient pas conscients du fait que les chefs d'établissement, les enseignants et les élèves ne disposaient peut-être pas des compétences nécessaires pour atteindre ces objectifs. Et surtout, les enseignants n'avaient peut-être pas les compétences suffisantes pour diagnostiquer les difficultés d'apprentissage, ni n'avaient accès à un répertoire suffisamment fourni de stratégies pédagogiques efficaces. D'un point de vue organisationnel, la culture de responsabilité pour garantir l'égal apprentissage de tous les élèves était parfois absente de ces établissements. Faisaient également défaut les processus de diffusion des pratiques de classe efficaces et l'utilisation adéquate des ressources et des séances de développement professionnel des enseignants.

Des dispositifs de nouvelle génération voient le jour au niveau du district, intermédiaire naturel entre les États et les établissements scolaires. Ces systèmes traduisent la volonté de coordonner les exigences d'obligation de résultats avec le renforcement local des compétences (voir par exemple Anderson \& Togneri, 2002 ; Center for Research on the Context of Teaching, 2002 ; Hightower, Knapp, Marsh et al., 2002 ; Supovitz, 2006). Les dispositifs mis en place par les districts sont conçus pour allier pression politique sur les établissements (afin de satisfaire aux objectifs annuels de progrès des élèves) et amélioration de l'accès aux ressources et à diverses formes d'accompagnement. Un « principe de réciprocité » guide cet équilibre (Elmore, 2004). La réussite du dispositif repose sur la qualité et l'accessibilité des ressources et, dans la durée, sur l'adaptabilité du système face aux nouveaux besoins des établissements scolaires, en fonction des nouvelles exigences de performance. Ces dispositifs sont de durée, de ressources, d'accompagnement, de qualité de mise en œuvre et d'efficacité variables. Ils reflètent tous la volonté d'une meilleure qualité de l'information disponible pour améliorer in fine l'enseignement. Ce dispositif comprend un temps de réflexion collective sur la qualité des pratiques pédagogiques, le recours aux évaluations pour suivre et diagnostiquer les apprentissages des élèves et l'accès au développement professionnel à partir des besoins pédagogiques. Les résultats de la recherche montrent que ce type de processus collaboratif et les ressources de formation des enseignants contribuent significativement à l'amélioration des performances des élèves (Bryk, Sebring, Allensworth et al., 2010 ; Carroll, Fulton \& Doerr, 2010).

La récente réforme du système éducatif de la ville de New York, Children First, donne un aperçu de la façon dont les responsables politiques peuvent allier obligation de résultats et renforcement des capacités d'adaptation des établissements à leur environnement. La ville de New York abrite le plus grand système d'écoles publiques aux États-Unis : 1600 établissements scolaires, 80000 enseignants et 1,1 million d'élèves, dont un grand nombre issu de milieux défavorisés et dont l'anglais n'est pas la langue première. À l'automne 2007, la majeure partie de ce dispositif était en place. Sont exposés ici les grandes lignes du plan Children First, notamment les intentions exprimées par les responsables politiques d'aligner les ressources disponibles sur les objectifs fixés. Cet article se base sur les résultats d'une étude qualitative sur le pilotage du processus d'enquête collaborative dans tous les établissements scolaires de la ville (Robinson, Kannapel, Gujarati et al., 2008). Ce processus d'enquête, pierre angulaire du dispositif, vise, sur un plan stratégique, à mieux coordonner attentes externes, qualité de l'enseignement et répartition interne des ressources. L'analyse de la façon dont les établissements mettent à profit ce processus permet de repérer jusqu'à quel point telle ou telle partie du dispositif contribue aux besoins de renforcement local des capacités collectives. La mise en avant des points forts et des points faibles de ce processus permet aux responsables politiques de déterminer les choix stratégiques à opérer lorsqu'ils doivent concevoir des systèmes d'obligation de résultats qui stimulent et accompagnent le développement des établissements scolaires.

\section{CHOIX STRATÉGIQUES ET RÉFORME DU SYSTÈME SCOLAIRE}

Au cours de l'année scolaire 2007-2008, le ministère de l'éducation de la ville de New York a lancé la seconde phase de Children First. Ce plan devait 
transformer la culture et le fonctionnement des structures administratives du district et des établissements scolaires. Selon les responsables politiques, la restructuration du système éducatif a été guidée par trois principes : le leadership, la responsabilisation et l'obligation de résultats. Ce nouveau plan associe obligation de résultats et ressources supplémentaires afin de renforcer les capacités des établissements scolaires. L'obligation de résultats est marquée par la définition d'objectifs chiffrés de performance, la diffusion publique du rapport annuel de l'établissement et la responsabilité locale des équipes éducatives quant aux progrès des apprentissages des élèves. Le soutien comprend l'accès des établissements à un accompagnement spécialisé et des ressources supplémentaires pour développer les connaissances et les compétences nécessaires. Par ailleurs les chefs d'établissement et les enseignants sont désormais autorisés à prendre des initiatives selon les besoins spécifiques de leurs élèves.

\section{Dispositions communes à tout le système}

La mise en œuvre du programme comprend un certain nombre de dispositions qui imposent des objectifs de performance et donnent accès à de nouvelles formes d'accompagnement afin de renforcer les capacités individuelles et de l'organisation des établissements. II s'agit, entre autres, d'améliorer l'accès des établissements à des informations pertinentes sur leurs élèves, le développement professionnel lié à l'enseignement et au leadership et une orientation de qualité des élèves, le tout assuré par des enseignants et des administrateurs reconnus.

\section{Retour d'information sur les performances de chaque élève}

Les responsables politiques publient chaque année un bilan des performances de chaque école pour attirer l'attention des chefs d'établissement et des enseignants sur les progrès de leurs élèves. Ces rapports publics portent sur les progrès des établissements (ou school progress reports) et leur qualité (ou school quality reviews). Ils indiquent les résultats de chaque élève, apportent des informations sur la culture de l'établissement, le leadership et les processus de gestion des données. L'articulation entre les informations sur les performances des élèves et les processus organisationnels vise à encourager la focalisation de l'établissement sur les apprentissages des élèves. Ces rapports détaillent également la performance des élèves par rapport aux objectifs fixés. Chaque rapport comprend une analyse de la contribution de l'établissement à la performance des élèves, notamment les progrès réalisés par les élèves les plus en difficulté et ceux de quelques sous-groupes clés selon la matière, le genre, le niveau social et l'origine ethnique. Les établissements sont notés d'A à $F$ selon une batterie d'indicateurs, dont les progrès des élèves calculés en fonction des performances longitudinales des sous-groupes (60\%), les performances annuelles des élèves par sous-groupe (25\%), l'environnement scolaire et les progrès réalisés dans la réduction des écarts de performance (15\%). Les établissements qui n'atteignent pas les objectifs de performance doivent compléter leurs services éducatifs (tutorat, remédiation, notification aux parents de la possibilité de scolariser leurs enfants dans d'autres établissements publics par exemple). Si l'établissement n'atteint pas les objectifs fixés au bout de cinq ans, le ministère procède à des changements dans la gestion de l'établissement.

Les bilans de qualité (ou quality reviews) assurent un retour d'information régulier aux établissements sur la qualité de leur culture organisationnelle et sur les processus de gestion des informations, essentiels à l'amélioration des apprentissages des élèves. Chaque rapport dresse un profil de l'établissement suite à une visite de plusieurs jours effectuée par des responsables reconnus. Au cours de ces visites, ils rencontrent le personnel, les élèves et les parents; ils assistent à des cours et à des réunions professionnelles, étudient la façon dont les enseignants produisent et utilisent les informations sur les apprentissages des élèves pour améliorer les projets et les pratiques de l'établissement. Après chaque rapport, chaque établissement reçoit une appréciation qui va d'« insuffisant » à « satisfaisant ».

\section{Un nouveau marché pour l'accompagnement scolaire}

La mise en place d'un nouveau marché de prestations de services au sein duquel les établissements scolaires se positionnent en tant que consommateurs avertis est la décision la plus marquante de cette réforme. Ce marché a pour fonction d'assurer le contrôle qualité et de garantir la bonne adéquation entre les besoins des établissements scolaires et les ressources disponibles destinées à l'amélioration des résultats des élèves. II est constitué d'organismes publics et privés de soutien aux établissements (school support organisations, appelées SSO par la suite), chargés de fournir des prestations sur mesure relatives au curriculum, au développement professionnel et au leadership. Ces organismes doivent être réactifs face à l'évolution des besoins des établissements. Le 
ministère de l'Éducation valide ou recale les candidatures à ce type de statut selon leur conformité aux objectifs prédéfinis. Ces organismes se retrouvent alors en concurrence deux fois par an pour décrocher des contrats auprès des chefs d'établissement. Ils proposent des bouquets de services personnalisables adaptés aux objectifs déterminés par les chefs d'établissement. Les écoles membres sont organisées en réseaux de 20 établissements par les SSO pour faciliter l'apprentissage collaboratif autour d'objectifs d'amélioration communs.

\section{Informations sur la qualité de l'enseignement et des apprentissages}

L'amélioration de l'accès des établissements aux informations sur l'enseignement et les apprentissages est au cœur de la réforme. Les responsables politiques ont apporté deux ressources supplémentaires afin d'orienter les investissements des établissements en renforcement des capacités. La première consiste en un système global de gestion de données (achievement reporting and innovation system, ARIS ci-dessous). À partir d'une seule entrée, ce système permet d'obtenir des informations longitudinales sur l'origine démographique des élèves, les effectifs, le contrôle continu, les notes, l'assiduité, la discipline, les résultats, etc. Cet outil permet aux administrateurs et aux enseignants d'analyser la performance des élèves selon un ensemble de facteurs ; il s'agit ainsi d'encourager la collaboration et le partage d'informations entre enseignants par des bibliothèques de ressources électroniques et des domaines d'échanges, au sein d'un même établissement et d'un établissement à un autre.

La seconde ressource est la mise à disposition des enseignants de portefeuilles d'évaluations qui offrent des informations détaillées et opportunes sur les forces et les faiblesses de leurs élèves. Ces évaluations formatives aident les enseignants dans le suivi et le diagnostic des progrès de leurs élèves, dans leur réflexion sur l'efficacité de leurs pratiques pédagogiques et dans leur recherche de ressources. Ces évaluations sont disponibles en mathématiques et en anglais tout au long de l'année. Des séances de développement professionnel sont prévues pour accompagner les enseignants dans l'utilisation et l'analyse des résultats. Les résultats sont disponibles rapidement sur Internet ; ils peuvent être analysés selon différentes perspectives pour faciliter l'analyse des acquis d'élèves dans tel ou tel domaine. Afin d'encourager l'utilisation de ces évaluations par les enseignants, les responsables politiques se sont engagés publiquement à ne pas faire usage des résultats des évaluations formatives à des fins d'accountability.

\section{Assistance technique}

De nouveaux postes spécialisés ont été créés pour accompagner la formation des enseignants et l'amélioration des établissements. Ces postes doivent faciliter l'utilisation des données par les enseignants et les orienter au mieux parmi l'ensemble des données disponibles. Un "spécialiste des données " chargé de promouvoir leur utilisation a été nommé dans chaque établissement afin de mieux éclairer les choix pédagogiques des enseignants et la répartition des ressources. Sa mission consiste à aider les enseignants et les administrateurs à maîtriser les nouvelles ressources présentes dans le système ARIS. Ces spécialistes renseignent aussi les enseignants sur les points clés des évaluations formatives et les aident à interpréter les résultats. Symboliquement, la création de ce type de poste marque la valeur que les responsables publics accordent aux apprentissages des élèves.

Des « facilitateurs 》 (student achievement facilitators), responsables éducatifs confirmés, accompagnent également les établissements. Ces « facilitateurs » discutent in situ avec les équipes pédagogiques et les administrateurs des performances des élèves et les aident à interpréter le profil de chaque élève selon ses acquis, pour une meilleure préparation des séquences d'apprentissage. Ils informent aussi les établissements sur la parution et l'accès à de nouvelles ressources. Tout comme le personnel du ministère, ils travaillent en étroite collaboration avec le personnel des organismes de soutien aux établissements (SSO) pour faire le point sur les dernières politiques éducatives et les ressources disponibles.

\section{Dispositions propres à chaque établissement}

Le programme Children First comprend des mécanismes qui font basculer le contrôle et la responsabilité des ressources sur les chefs d'établissement et qui, par un processus collaboratif, permettent aux établissements scolaires de produire de nouvelles connaissances sur les besoins des élèves, les conditions d'enseignement et d'apprentissage à l'école et les pratiques efficaces.

\section{Responsabilisation des chefs d'établissement}

Les responsables publics ont établi de nouveaux contrats de performance qui rendent les chefs d'établissement responsables des gains annuels de 
performance des élèves. En contrepartie de cette charge supplémentaire, il leur a été confié la gestion des ressources de l'établissement, notamment la maîtrise du budget et l'embauche des enseignants. Ils gèrent aussi le contrat de service avec les organismes de soutien aux établissements et peuvent à tout moment mettre un terme au contrat s'ils n'en sont pas satisfaits. Une fois par trimestre, le ministère leur demande s'ils sont satisfaits de la qualité des «facilitateurs » et des organismes de soutien aux établissements. En fonction des performances de leur institution, les chefs d'établissement peuvent obtenir une prime, ou à l'inverse être démis de leurs fonctions.

\section{Enquête collaborative}

Le projet Children First encourageait aussi le développement individuel et de l'organisation des établissements par l'adoption de la méthode d'enquête collaborative dans toutes les écoles de la ville. En 2007, chaque école devait mettre en place une "équipe d'enquête " composée d'enseignants et d'administrateurs chargés d'analyser les problèmes d'apprentissage d'un petit nombre d'élèves en difficulté. Par ce processus d'enquête, les enseignants conçoivent et testent de nouvelles stratégies pédagogiques sur telle ou telle compétence ou tel ou tel concept qui a pu constituer un frein à l'apprentissage de leurs élèves. Ces derniers figurent parmi les plus faibles de l'école et sont ceux sur lesquels portent les efforts pour réduire les écarts de réussite avec les autres élèves. Les enseignants doivent recourir aux évaluations précédentes des élèves et à d'autres informations susceptibles d'orienter leurs choix pédagogiques.

L'enquête collaborative a pour objectif d'aider les équipes à partager et à développer leurs connaissances dans deux domaines jugés essentiels à une meilleure qualité de l'enseignement (Cochran-Smith \& Lytle, 1999). II s'agit tout d'abord d'affiner l'analyse des pratiques de classe actuelles sur la compétence ou le concept ciblé. Désignée sous le nom de " connaissance des pratiques ", l'enquête comprend l'examen par les équipes de l'application des programmes scolaires dans les classes, des choix pédagogiques et évaluatifs des enseignants et de leurs convictions quant à l'acquisition ou non de la compétence ou du concept étudié. Ces connaissances émergent lors des premières phases de l'enquête collaborative et permettent aux enseignants d'identifier les différences de pratiques pédagogiques et les omissions du programme, facteurs susceptibles de freiner l'apprentissage des élèves. Le second domaine, « connaissances au service des pratiques », porte sur la question de savoir comment et pourquoi certaines pratiques de classe sont plus efficaces que d'autres pour répondre aux besoins des élèves en difficulté. Ces informations proviennent des échanges avec les enseignants dont les pratiques sont efficaces pour un certain type d'élèves, ou alors des recherches externes sur les bonnes pratiques. Cet apport d'informations influence les réponses pédagogiques apportées par les équipes d'enquête, dont on attend qu'elles partagent ces nouvelles connaissances avec l'ensemble des enseignants et des administrateurs. On les encourage également à faire des propositions de formation professionnelle pour aider les enseignants à s'approprier ces nouvelles pratiques. L'enquête collaborative est donc un processus sur lequel les responsables scolaires peuvent s'appuyer pour déterminer les compétences que les enseignants doivent acquérir pour répondre à des besoins spécifiques.

À des moments clés du processus (voir la figure 1), les enseignants doivent s'appuyer sur le nouveau dispositif pour guider leurs choix. Les équipes sont alors censées avoir une bonne maîtrise du processus d'enquête lui-même et des outils de diagnostic pour déterminer les domaines sur lesquels l'établissement scolaire doit mettre l'accent. Les spécialistes des données, les «facilitateurs » et les organismes de soutien aux établissements doivent aussi accompagner les équipes. D'autres ressources comme, au niveau matériel, des ordinateurs portables et des heures supplémentaires, complètent le travail d'équipe. Au fil du temps, chaque établissement doit partir de l'équipe pilote et multiplier les équipes d'enseignants pour étendre le processus d'enquête à au moins $90 \%$ du personnel. La généralisation de l'engagement dans ce processus doit promouvoir une culture de la responsabilité dans tout l'établissement et le recours à des méthodes ayant fait leurs preuves pour relever la qualité de l'enseignement et des apprentissages au sein de l'établissement.

Les décisions des responsables publics reflètent une stratégie visant à coordonner objectifs de performance et apport de nouvelles ressources dédiées à l'accompagnement des besoins spécifiques des élèves. Ces outils focalisent l'attention des chefs d'établissement et des enseignants sur les résultats des élèves, notamment ceux des plus défavorisés. En lieu et place d'une même méthode pour tous les établissements, il est laissé à la discrétion des chefs d'établissement et des enseignants le soin de déterminer les connaissances et les compétences nécessaires 
Figure 1. Production et utilisation des connaissances par l'enquête collaborative lors de l'année scolaire 2007-2008

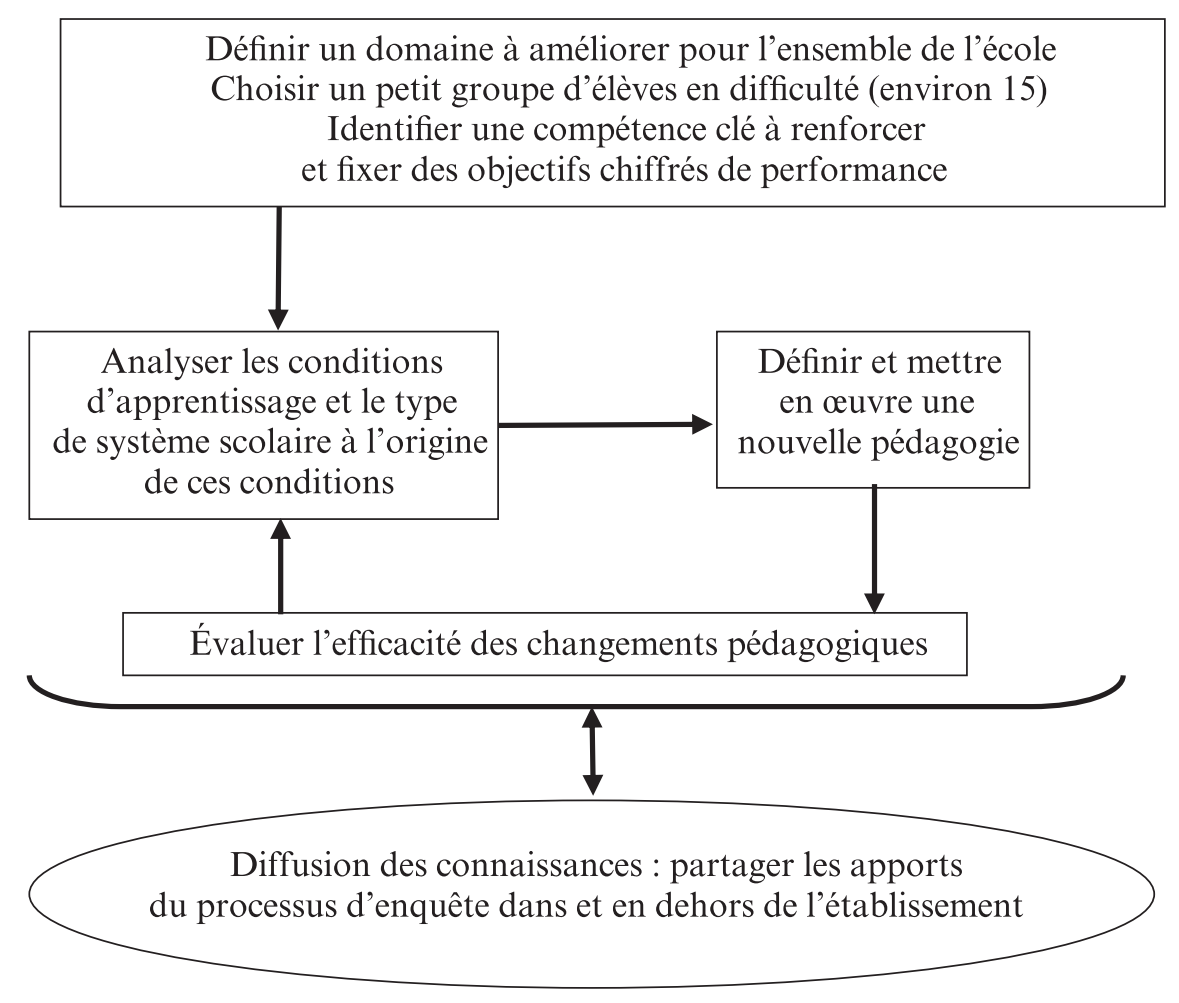

au personnel de l'établissement pour parvenir à cet objectif et de mettre en place les formations professionnelles et les ressources utiles à cet effet.

\section{ANALYSE DE LA RÉFORME SUR LE TERRAIN}

Au niveau de la conception, les liens entre obligation de résultats et renforcement des capacités collectives ou, en d'autres termes, entre pression et soutien semblent cohérents. Dans quelle mesure cette cohérence se reflète-t-elle dans les pratiques de terrain ? II faudra du temps et de nombreuses recherches pour répondre avec certitude à cette question. En attendant, l'engagement des établissements dans le processus d'enquête collaborative offre un bon aperçu de cette cohérence. Rappelons qu'il s'agit d'un dispositif mis sur pied par les responsables politiques pour renforcer les capacités et compétences des acteurs de terrain. Les objectifs affichés et le processus lui-même pointent certaines des compé- tences que les établissements doivent développer pour atteindre les nouveaux objectifs de performance. II s'agit d'encourager les enseignants à utiliser les données disponibles pour faire le diagnostic des difficultés des élèves et trouver la bonne remédiation ; développer des procédures organisationnelles pour partager les nouvelles connaissances des enseignants ; utiliser ces connaissances pour adapter le projet d'établissement et la répartition des crédits ; et encourager la responsabilisation collective du personnel au service de la performance des élèves.

Jusqu'à quel point l'enquête collaborative aide-telle les établissements à utiliser les nouvelles ressources et supports disponibles, à acquérir de nouvelles compétences pour faire face aux difficultés des élèves les moins performants ? L'analyse présentée ici est le fruit d'une étude qualitative effectuée en 2008 sur la mise en œuvre de cette méthode, un an après son lancement, dans tous les établissements scolaires de la ville de New York. L'échantillon de l'enquête est composé de 41 établissements 
publics - 2 établissements d'enseignement spécialisé et un échantillon aléatoire de 39 établissements d'enseignement général. Les données proviennent de 243 entretiens auprès des équipes d'enquête et des chefs d'établissement et de l'observation de 34 réunions d'équipes. La taille des équipes allait de 3 à 12 membres. Les entretiens portaient sur le contexte de l'établissement, les objectifs, le processus et les résultats de l'enquête collaborative, l'accompagnement et les ressources essentielles, et l'utilisation par les équipes des résultats en vue d'améliorer l'établissement. Les observations d'équipes étaient centrées sur l'utilisation des données et la dynamique d'équipe. Pour synthétiser les informations recueillies, des profils descriptifs de chaque établissement ont été établis, dans lesquels figurent les caractéristiques principales et les résultats du processus d'enquête, ainsi que la contribution de ce processus au renforcement des compétences du personnel. Les données issues des entretiens et des observations et les profils des établissements ont été codés avec Atlas.ti, logiciel de gestion de données, afin de faciliter l'analyse.

\section{Rythme de mise en œuvre \\ et utilisation des éléments clés du dispositif}

Avant d'analyser les apports du processus d'enquête dans le renforcement des compétences au sein des établissements, il convient de déterminer son rythme de mise en œuvre et de repérer les éléments du dispositif qui ont influencé le travail d'enquête. Tous les établissements de l'étude avaient mis sur pied une équipe d'enquête et la grande majorité d'entre eux avait concentré ses efforts sur les élèves les plus en difficulté. La phase de démarrage de ce processus a été lente et le rythme irrégulier, puisque les équipes se familiarisaient avec le processus et découvraient la diversité des ressources et données disponibles. Environ les trois quarts des établissements ont pu mener le processus à son terme (voir la figure 1), à savoir du diagnostic des problèmes d'apprentissage à la formulation de recommandations pour améliorer l'établissement. Les enseignants concernés estimaient que le processus était en phase avec les objectifs de performance et les ressources disponibles. Ils ont fait état de nouvelles perspectives sur les conditions d'enseignement et d'apprentissage qui, tout comme les stratégies pédagogiques, devaient faire l'objet de certains ajustements dans leur établissement pour mieux accompagner les élèves en difficulté. Dans l'ensemble, une majorité estimait que le processus d'enquête était utile à leur développement professionnel et à leur établissement et était impatiente de renouveler l'expérience l'année suivante.

Le tableau 1 dresse la liste des éléments du dispositif et leur fréquence d'utilisation selon les équipes. Seules deux ressources ont posé problème : l'accès à la base de données ARIS était peu fiable en raison de problèmes techniques ; les équipes n'ont par ailleurs guère fait appel à l'expertise des organismes privés de soutien aux établissements, par méconnaissance de leurs services. Les entretiens avec les chefs d'établissement ont néanmoins montré que ces organismes ont pour la plupart assuré des prestations auprès des établissements mais non auprès des équipes d'enquête. Le personnel de ces organismes pensait que d'autres personnes du ministère étaient responsables de l'accompagnement des équipes. La partie suivante analyse la façon dont le processus d'enquête collaborative contribue à l'amélioration des apprentissages chez les élèves et de l'organisation des établissements.

\section{Identifier les nouvelles connaissances et compétences nécessaires au développement professionnel des enseignants}

Au cœur du renforcement des compétences pour améliorer les acquis des élèves, notamment dans des domaines où l'établissement a toujours été en échec, figure le développement des connaissances et compétences des enseignants. Les enseignants ont identifié trois domaines dans lesquels leurs connaissances s'étaient enrichies suite au travail d'enquête : compréhension et usage des évaluations pour diagnostiquer le niveau des élèves et suivre leur apprentissage ; pratiques pédagogiques inhibitrices ; nouvelles stratégies pédagogiques efficaces. La plupart des équipes ont fait des progrès considérables dans la compréhension et l'usage des évaluations suite au travail d'enquête. Ces progrès sont dus en partie aux options choisies pour le processus d'enquête et aux récentes évaluations formatives. Le tableau 2 indique les diverses sources d'information sur les résultats des élèves, l'environnement de l'établissement et les réponses pédagogiques sur lesquelles les équipes se sont appuyées pour leur travail d'enquête. Les équipes comptaient sur les spécialistes du traitement de données et les « facilitateurs" pour favoriser l'accès aux données et leur interprétation. Ces intervenants avaient le mérite d'enrichir la qualité des conversations et de permettre d'accéder à de nouvelles ressources d'évaluation. 
Tableau 1. Utilisation des supports au service de l'enquête collaborative (année scolaire 2007-2008)

\begin{tabular}{|c|c|c|}
\hline Supports & Types de ressources et d'accompagnement & $\begin{array}{l}\text { Fréquence d'utilisation } \\
\text { par les équipes d'enquête }\end{array}$ \\
\hline \multirow{2}{*}{ Outils d'obligation de résultats } & Rapports sur les progrès des établissements & Élevée \\
\hline & Bilans de qualité & Élevée \\
\hline \multirow{3}{*}{ Accès aux données et formation } & ARIS & Modérée \\
\hline & Évaluations périodiques et/ou formatives & Élevée \\
\hline & Données issues des évaluations de l'État & Élevée \\
\hline Personnel spécialisé & $\begin{array}{l}\text { Spécialistes des données au sein des } \\
\text { établissements et "facilitateurs" }\end{array}$ & Élevée \\
\hline \multirow{2}{*}{ Collaboration en équipe } & Heures supplémentaires & Élevée \\
\hline & Ordinateurs portables & Élevée \\
\hline \multirow{2}{*}{$\begin{array}{l}\text { Organisme externe de soutien } \\
\text { aux établissements (ou SSO) }\end{array}$} & $\begin{array}{l}\text { Ressources pédagogiques et développement } \\
\text { professionnel }\end{array}$ & Faible, voire aucune \\
\hline & Personnel au service des établissements & Faible, voire aucune \\
\hline
\end{tabular}

Tableau 2. Types de données utilisées par les équipes d'enquête (année scolaire 2007-2008)

\begin{tabular}{|c|c|}
\hline Décisions du processus d'enquête & Données utilisées \\
\hline $\begin{array}{l}\text { Déterminer un domaine d'amélioration commun à tout } \\
\text { l'établissement }\end{array}$ & $\begin{array}{l}\text { Outils d'obligation de résultats } \\
\text { Évaluations de l'État } \\
\text { Programmes scolaires } \\
\text { Domaines négligés au sein de l'établissement }\end{array}$ \\
\hline Choisir un petit groupe d'élèves comme objet d'étude & $\begin{array}{l}\text { Évaluations de l'État } \\
\text { Dossiers des élèves et/ou de la classe dans ARIS } \\
\text { Échanges avec les enseignants, les élèves } \\
\text { et les conseillers d'orientation }\end{array}$ \\
\hline Diagnostiquer les besoins des élèves sélectionnés & $\begin{array}{l}\text { Évaluations formatives } \\
\text { Observations de classe } \\
\text { Dossiers scolaires } \\
\text { Entretiens avec les élèves et les enseignants } \\
\text { Travail des élèves }\end{array}$ \\
\hline $\begin{array}{l}\text { Élaborer et mettre en place une nouvelle stratégie } \\
\text { pédagogique }\end{array}$ & $\begin{array}{l}\text { Recommandations des membres de l'équipe } \\
\text { Programmes scolaires existants } \\
\text { Expertise des «facilitateurs» } \\
\text { Publications professionnelles }\end{array}$ \\
\hline Évaluer l'efficacité de cette nouvelle stratégie & $\begin{array}{l}\text { Évaluations formatives } \\
\text { Observations } \\
\text { Travail des élèves } \\
\text { Évaluations de l'État } \\
\text { Dossiers scolaires }\end{array}$ \\
\hline
\end{tabular}


Grâce à l'enquête collaborative, les équipes ont acquis une meilleure connaissance des données disponibles et de la valeur de chaque source pour orienter leur pédagogie. Elles étaient aussi plus à l'aise pour trouver les sources appropriées, recueillir des informations sur les élèves et leurs processus d'apprentissage et identifier leurs points faibles. Par exemple, les équipes ont analysé les forces et les faiblesses de différentes évaluations formatives et leur capacité à mesurer leurs acquis dans tel ou tel domaine. Les échanges entre enseignants leur ont permis d'interpréter les résultats des évaluations et de s'en servir comme base de départ pour leurs choix pédagogiques.

Les équipes ont aussi mieux appréhendé les conditions d'enseignement et d'apprentissage au sein de l'établissement. Le processus d'enquête s'est penché sur la façon dont les compétences et concepts ciblés étaient enseignés, d'où une remise en cause des pratiques pédagogiques à l'origine des freins à l'apprentissage. Des observations de cours et l'analyse de la relation entre les besoins des élèves en difficulté et les pratiques pédagogiques du moment ont contribué à l'émergence de ces connaissances. Par exemple, un établissement s'est aperçu que son choix d'évaluation formative pour suivre les progrès des élèves en lecture ne mesurait pas les compétences essentielles en lecture, d'où une méconnaissance des enseignants sur un aspect important de l'apprentissage dans ce domaine. Dans un autre établissement, des incohérences dans la mise en œuvre du programme de lecture ont réduit son efficacité. Les bibliothèques n'étaient pas correctement organisées et n'offraient qu'un choix limité de livres pour les élèves les plus faibles. Les équipes d'enquête ont également remarqué que certains enseignants faisaient passer le test de lecture interne de façon inégale. Certains n'évaluaient pas la lecture chaque mois comme prévu et d'autres ne faisaient passer qu'une partie du test. Dans un troisième établissement, une mauvaise coordination entre le curriculum et le dispositif de tutorat expliquait en partie les progrès insuffisants des élèves. Le processus d'enquête collaborative a permis de faire émerger de précieuses informations sur les pratiques enseignantes et les conditions de travail en classe à l'origine des freins aux apprentissages.

Le troisième domaine d'acquisition de connaissances des enseignants portait sur le repérage des stratégies pédagogiques efficaces. Les enseignants ou les conseillers pédagogiques des équipes d'enquête arrêtaient leurs stratégies à partir de l'analyse des matériaux existants dans l'établissement ou des échanges avec les enseignants qui étaient rompus à l'accompagnement des élèves avec les mêmes besoins d'apprentissage que les groupes étudiés. Les stratégies retenues portaient sur la gestion de classe et la mise en place d'une pédagogie différenciée. Certaines stratégies pédagogiques se contentaient d'un ajustement des pratiques pédagogiques en cours à un public spécifique ou à des élèves issus de l'enseignement spécialisé.

\section{Partager de nouvelles connaissances dans et hors de l'établissement}

Il est nécessaire que les établissements développent des procédures visant à diffuser les connaissances acquises auprès de l'ensemble du personnel. À cette fin, les équipes sont invitées à partager leurs expériences et leurs impressions avec d'autres collègues pour éclairer la valeur de leurs décisions. Le partage des résultats de l'enquête permet par ailleurs d'élargir les discussions sur les domaines où les progrès sont nécessaires. Au cours de la première année de mise en place du dispositif, de nombreuses équipes ont rencontré d'autres enseignants au sein de leur établissement, pour raconter le déroulement du processus d'enquête, leur usage des données et la stratégie pédagogique retenue. De nombreuses équipes ont aussi mis en place des réunions informelles pour partager leurs connaissances lors de petits-déjeuners ou déjeuners, des invitations à venir assister à des réunions des équipes d'enquête ou la distribution de tracts dans les casiers des enseignants. Lors des échanges interétablissements, rares ont été les équipes à utiliser ARIS pour contacter leurs collègues qui travaillent sur les mêmes problèmes d'apprentissage. Seuls quelques réseaux SSO ont accueilli des manifestations pour faciliter le partage des résultats entre établissements. Les équipes ont presque toutes souhaité faire partager leurs expériences mais n'osaient pas franchir le pas car elles étaient toujours en phase d'apprentissage. Elles étaient aussi nombreuses à préférer attendre la confirmation de leur démarche par les résultats des élèves lors de l'évaluation d'État qui se déroulait souvent en fin d'année scolaire. Si les procédures existantes contribuaient au partage d'informations générales, elles ne favorisaient guère la réflexion sur le sens et la valeur des résultats du processus d'enquête. Ces résultats soulignent néanmoins les limites du recours à une seule équipe d'enquête pour la production de connaissances et leur diffusion dans l'établissement. Les réponses des enseignants montrent 
que ce travail d'enquête est amené à être davantage partagé, dans la mesure où un plus grand nombre d'enseignants pourrait être directement impliqué dans le processus.

\section{Utiliser ces nouvelles connaissances pour améliorer l'organisation de l'établissement}

Les établissements doivent mettre ces nouvelles connaissances au service du développement individuel et au niveau de l'organisation des établissements. Plus précisément, ils doivent s'appuyer sur les résultats de l'enquête collaborative pour nourrir les choix de développement professionnel et apporter les ajustements systémiques au projet d'établissement (curriculum et répartition des ressources au service de nouvelles pratiques). Dans certains établissements, les équipes parrainaient des collègues pour qu'ils se forment dans deux domaines précis. II s'agissait tout d'abord d'aider les enseignants à comprendre et utiliser les nouvelles évaluations formatives pour diagnostiquer et suivre les progrès des élèves. Ces ateliers concernaient tous les enseignants, étaient obligatoires et faisaient l'objet d'une journée officielle de formation professionnelle. Un expert externe animait la formation. Le second domaine portait sur la stratégie pédagogique qui, selon les équipes d'enquête, avait fait ses preuves pour répondre aux besoins des élèves ciblés. Ces ateliers étaient courts (30 à 60 minutes), sur la base du volontariat, avaient lieu pendant le temps de travail et étaient animés par les équipes d'enquête qui avaient mis en œuvre la stratégie.

Dans la plupart des équipes, certains estimaient que l'objectif suprême du processus d'enquête était d'apporter des changements systémiques au sein de leur établissement. Néanmoins seule la moitié des équipes a formulé des recommandations pour adapter le projet d'établissement et modifier éventuellement le curriculum. Les recommandations qui tendaient à améliorer l'efficacité des procédures et des ressources existantes étaient apparemment mises en œuvre plus rapidement. En revanche, celles qui portaient sur les nouvelles stratégies pédagogiques n'étaient pas intégrées formellement dans le curriculum. Lorsque ces stratégies étaient partagées, c'était toujours de façon informelle et ce partage était laissé à la discrétion des enseignants. L'enquête a débouché sur toute une série d'initiatives au fur et à mesure que les équipes analysaient les problèmes d'apprentissage des élèves et évaluaient les conditions d'enseignement et d'apprentissage. Ces initiatives dépen- daient des résultats de l'enquête, en particulier de la valorisation des évaluations formatives pour éclairer les pratiques de classe et l'efficacité des stratégies pédagogiques.

\section{Responsabiliser l'ensemble des équipes au service des apprentissages des élèves}

Le processus d'enquête collaborative s'avère prometteur pour aider les établissements à responsabiliser l'ensemble du personnel autour de la réussite des élèves. L'approche pas à pas - ciblage d'un seul problème d'apprentissage important, connaissance progressive d'un petit groupe d'élèves en difficulté, travail intensif avec une équipe restreinte d'enseignants - a largement contribué à faire du processus d'enquête un outil pertinent et productif. Les équipes d'enquête ont indiqué que le travail en étroite collaboration avec un petit groupe d'élèves renforçait leur motivation pour assurer leur réussite. Nombre d'enseignants appréciaient ce processus parce qu'ils y voyaient le reflet de leur motivation première lorsqu'ils sont entrés dans le monde de l'éducation : améliorer le sort des classes en général et celui de leurs élèves en particulier. Enfin c'était l'occasion pour les administrateurs, débordés par les problèmes disciplinaires et les questions d'entretien des bâtiments, de s'intéresser davantage à la vie des classes et d'échanger avec leurs équipes enseignantes. L'engagement dont font preuve les équipes vis-à-vis des progrès d'apprentissage des élèves ciblés est une réussite incontestable, un an après la mise en place de ce processus. Néanmoins, l'implication de l'ensemble du personnel de l'établissement nécessite l'engagement d'un plus grand nombre d'enseignants et d'administrateurs dans le processus et le soutien des chefs d'établissement, pour faire de la réussite des élèves l'objectif de tous. Pour responsabiliser l'ensemble de l'équipe éducative, le processus d'enquête devra être intégré dans l'organisation même de l'établissement et rallier un plus grand nombre d'enseignants aux groupes de travail existants.

\section{CHOIX STRATÉGIQUES D'OBLIGATION DE RÉSULTATS}

Notre analyse globale du processus d'enquête collaborative souligne à quel point l'articulation entre obligation de résultats et apport de ressources supplémentaires peut contribuer à renforcer les 
compétences des établissements. Elle révèle également quelques dysfonctionnements. Plus précisément, un meilleur fonctionnement du processus d'enquête collaborative passe par une clarification du rôle et des responsabilités des responsables des organismes de soutien aux établissements et de leur personnel, dans le cap à tenir pour améliorer le fonctionnement des établissements. L'accès des équipes à l'expertise et aux ressources des SSO pour le montage des séquences pédagogiques contribuera à une meilleure qualité des échanges et des recommandations auprès des établissements.

D'autres choix stratégiques s'offrent aux responsables politiques pour accélérer les progrès dans tous les établissements. Par exemple, les résultats de l'étude montrent que la plupart des équipes d'enquête se sont focalisées sur les problèmes d'apprentissage des élèves en anglais et ont testé de nouvelles méthodes pour répondre à ces problèmes. II n'existait cependant aucun processus clair pour identifier les problèmes d'apprentissage communs à tous les établissements et pour voir si les ressources dont disposaient les établissements étaient susceptibles de répondre à ces besoins. Manquaient également la synthèse des acquis des enquêtes collaboratives ainsi qu'une méthode d'évaluation de leur qualité, qui aurait été nécessaire pour envisager une large diffusion. Ces informations pourraient guider l'enquête ou accélérer la planification pédagogique. Les organismes de soutien aux établissements (SSO) pourraient quant à eux réorienter leur offre de services ou leur aide technique auprès des établissements. Le tout fournirait une base incontestable de partage entre établissements.

La réforme Children First éclaire les choix stratégiques à opérer face au double objectif d'équité et d'excellence dans l'enseignement public. II s'agit dès la conception du dispositif d'allier objectifs de performance et investissements dans la capacité des établissements à satisfaire ces attentes. Une fois que le dispositif est mis en œuvre, de nouvelles occasions de renforcer et d'étendre cette alliance se font jour au fur et à mesure que les acteurs du système relèvent le défi d'améliorer la qualité de l'enseignement et des apprentissages.

Marian Robinson mrobinson@exchange.tc.columbia.edu Teachers College, Université de Columbia, États-Unis

\section{BIBLIOGRAPHIE}

ANDERSON S. \& TOGNERI W. (2002). Beyond islands of excellence: What districts can do to improve instruction and achievement in all schools. Washington : Learning First Alliance.

BRYK A., SEBRING P., ALLENSWORTH E., LUPPESCU S. \& EASTON J. (2010). Organizing schools for improvement: Lessons from Chicago. Chicago: University of Chicago Press.

CARROLL T., FULTON K. \& DOERR H. (2010). Team-up for 21st century teaching and learning: What research and practice reveal about professional learning. Washington : National Commission on Teaching and America's Future.

CENTER FOR RESEARCH ON THE CONTEXT OF TEACHING (2002). Bay area school reform collaborative: Phase one (1995-2001) evaluation. Stanford University : Center for Research on the Context of Teaching. Disponible sur Internet à l'adresse : <http://www.stanford.edu/group/ CRC/publications_files/BASRC_Phase_I_Report.pdf> (consulté le 26 janvier 2011).

COCHRAN-SMITH M. \& LYTLE S. (1999). «Relationships of knowledge and practice: Teacher learning in communities ". Review of Educational Research in Education, vol. 24, $\mathrm{n}^{\circ} 1$, p. 249-305.
ELMORE R. (2004). "Conclusion: The problem of stakes in performance-based accountability systems ". In S. Fuhrman \& R. Elmore (dir.), Redesigning accountability systems for education. New York : Teachers College Press.

FUHRMAN S. \& ELMORE R. (2004). Redesigning accountability systems for education. New York : Teachers College Press.

HIGHTOWER A., KNAPP M., MARSH J. \& McLAUGHLIN M. (2002). School districts and instructional renewal. New York : Teachers College Press.

ROBINSON M., KANNAPEL P., GUJARATI J., WILLIAMS H. \& OETTINGER A. (2008). " A formative study of the implementation of the inquiry team process in New York city public schools: 2007-08 findings ». New York : Consortium for Policy Research in Education. Disponible sur Internet à l'adresse : <http://www.cpre. org/images/stories/cpre_pdfs/cfi_research_ report_2008.pdf> (consulté le 26 janvier 2011).

SUPOVITZ J. (2006). The case for district-based reform: Leading, building and sustaining school improvement. Cambridge : Harvard Education Press. 
